Revista Voluntas: Estudos sobre Schopenhauer - Vol. 3, Números 1 e 2 - $1^{\circ}$ e $2^{\text {o }}$ semestres de 2012 - ISSN: 2179-3786 - pp. 111-121.

\title{
Schopenhauer e la fondazione laica dell'etica
}

\author{
Schopenhauer e a fundação laica da ética
}

\author{
Fabio Ciracì \\ Professor na Università degli Studi del Salento (Lecce-Itália), \\ Secretário do "Centro interdipartimentale di ricerca su Arthur Schopenhauer e la sua scuola" \\ E-mail: fabio.ciraci77@gmail.com
}

\begin{abstract}
Riassunto: Con il presente saggio si intende presentare la fondazione laica della morale di Arthur Schopenhauer, mostrando come si articola il discorso morale schopenhaueriano e descrivendo le due condizioni indicate dal filosofo per giungere alla negazione della volontà di vita: quella ascetica-contemplativa del genio, riservata a pochi eletti, e quella della compassione, aperta alla maggior parte degli uomini. La predisposizione alla compassione è individuale ed innata, come il carattere da cui ha origine. Tuttavia, il compatire, là dove sia una inclinazione naturale rimasta in potentia, può essere suscitato anche dall'esperienza del proprio dolore, attraverso una "seconda e migliore navigazione", lo schopenhaueriano deuteros plous.
\end{abstract}

Parole chiave: Schopenhauer; Compassione; Laicità.

Resumo: Com o presente artigo pretende-se apresentar a fundação laica da moral de Arthur Schopenhauer, mostrando como o discurso moral schopenhaueriano se articula e descrevendo as duas condições indicadas pelo filósofo para o alcance da negação da vontade de vida: a ascética-contemplativa do gênio, reservada a poucos eleitos, e a compaixão, aberta à maior parte dos homens. A pré-disposição à compaixão é individual e inata, assim como o caráter do qual ela se origina. Todavia, o compadecer-se, algo entendido como uma inclinação natural que permanece in potentia, pode ser suscitado também pela experiência da própria dor, mediante uma "segunda e melhor navegação", o schopenhaueriano deuteros plous.

Palavras-chave: Schopenhauer; Compaixão; Laicidade.

Perché nessuna idolatria è buona e da Schopenhauer non si esce idolatri

(Ceronetti, La lanterna del filosofo, p. 154).

\section{Discussione}

1.1. Schopenhauer come educatore. Schopenhauer deve essere sicuramente ricordato come il fondatore della morale laica in senso proprio. Laica, innanzitutto, perché Schopenhauer, per primo fra i pensatori occidentali, pone come essenza del mondo la volontà, che si esprime come corporeità e come natura, in maniera opposta alle morali che sostengono il primato dello spirito e del trascendente. Laica, perché la morale schopenhaueriana non giustifica il male, né lo rende 
Revista Voluntas: Estudos sobre Schopenhauer - Vol. 3, Números 1 e 2 - $1^{\circ}$ e $2^{\circ}$ semestres de 2012 - ISSN: 2179-3786 - pp. 111-121.

dialettico, ma lo affronta e ne indaga la causa metafisica. Laica, perché essa non deriva da alcuna teodicea (etica classica) e non ipotizza o giustifica nessun aldilà e nessun dio personale (etica kantiana). Laica, ancora, perché la visione del mondo insegnata da Schopenhauer è immanentistica e parte dall'esperienza, e si confronta costantemente con la scienza. Laica, perché nega alle religioni la loro pretesa assolutezza, ma le interpreta come fenomeni storici ovvero come «metafisica dei popoli», come «veicolo mitico», e cioè come sistemi di credenze necessarie per giustificare ed accettare il male e il nonsense della vita. Laica, infine, perché Schopenhauer propone un'etica fondata sull'uomo, in quanto unico essere capace di discernimento, di contemplazione e di compassione.

Se da un lato quella di Schopenhauer si presenta come una "filosofia del disincanto", con la quale si mette fine ad ogni visione ottimistica e consolatoria della realtà, dall'altro lato la metafisica schopenhaueriana fonda una "morale del disinganno", che smaschera i motivi egoistici dell'agire umano e introduce una nuova visione morale del mondo, in cui si abbandona ogni giaculatoria spiritualistica o idealistica, in cui non si giustificano gli orrori del mondo, ma viene indagata la loro radice. Non sarebbe certo errato, pertanto, annoverare Schopenhauer, con Marx, Freud e Nietzsche, fra quei maestri della cosiddetta "scuola del sospetto".

2.1 La critica della morale kantiana. L'analisi schopenhaueriana muove dalla critica della morale di Kant. Innanzitutto, il filosofo del Mondo definisce l'imperativo categorico kantiano un «comodo cuscino» con il quale si è reso omaggio all'astratta ragione formale e non alla realtà dell'uomo concreto. Il richiamo kantiano alla legge, l'utilizzo lessicale del "tu devi [sollst]" luterano, secondo Schopenhauer provengono dalla formazione pietistica di Kant, e sarebbero rivelatori della radice teologica del discorso morale kantiano. Lo stesso concetto kantiano di dovere «trova la sua origine nella morale teologica e rimane un estraneo in quella filosofica fin tanto che non abbia presentato una credenziale valida in relazione all'essenza della natura umana a quella del mondo oggettivo». Se da un lato Kant, con la Dialettica trascendentale, ha sbarrato la strada ad ogni fondazione cosmologica di Dio, dall'altro lato egli ha sostenuto «una teologia sorretta soltanto dalla morale, anzi da essa proveniente» ${ }^{1}$, e cioè «mediante ipotesi non motivate» ${ }^{2}$. Per Schopenhauer, quindi, l'esistenza di una morale sub specie aeternitatis prodotta dall'imperativo categorico è una petitio principii di Kant, una tesi tutta da dimostrare. Inoltre, l'imperativo categorico non ci dice nulla sul fondamento della morale, esso è una "mera istruzione", indica una procedura, ci dice il

\footnotetext{
${ }^{1}$ Idem, p. 124.

${ }^{2}$ Idem, p. 176.
} 


\section{FABIO CIRACÌ}

come ma non fornisce il che, il fondamento. «Dunque - conclude il Saggio di Francoforte - anche la fondazione kantiana dell'etica [...] sprofonda davanti ai nostri occhi nel grande e forse incolmabile abisso degli errori filosofici, per essersi dimostrata un'ipotesi illecita e un mero travestimento della morale teologica $»^{3}$.

2.2. Schopenhauer però non si limita a criticare la filosofia morale di Kant, del quale invece accetta la divisione fra carattere intelligibile e carattere empirico. L'intento del filosofo del Mondo è quello di fornire una nuova prospettiva, a partire dall'empirico e dall'essenza dell'uomo. Dalla volontà universale, in quanto essenza irrazionale e cieca del mondo, Schopenhauer fa derivare la profonda «immoralità del genere umano», smascherando, quindi, la vera e principale causa dell'agire umano: «Lo stimolo principale e fondamentale nell'uomo come negli animali è l'egoismo, cioè l'anelito all'esistenza e al benessere». Schopenhauer non costruisce la sua visione metafisica del mondo per giustificare la sua visione morale, ma «il fondamento etico, qualunque esso sia, deve avere a sua volta appiglio e un sostegno in qualche metafisica, cioè in una data spiegazione del mondo e dell'esistenza in genere». Compito del fillosofo è anche quello di denunciare l'utilizzo improprio di alcuni concetti di origine religiosa, introdotti surrettiziamente ed utilizzati in maniera equivoca anche in campo filosofico. «Per il popolo - scrive Schopenhauer - la morale viene motivata con la teologia come espressa volontà di Dio. I filosofi invece, con poche eccezioni, si studiano accuratamente di escludere del tutto questa specie di motivazione, pur di evitarla, ricorrono piuttosto a motivi sofistici». Schopenhauer si sforza di mostrare come anche le religioni positive si servano della minaccia di una pena per dar vita al proprio ordinamento morale: «Si aggiunge la convinzione che un'azione morale prodotta da una minaccia di castigo e dalla promessa di ricompensa sarebbe più morale in apparenza che secondo verità». Il filosofo di Danzica mette quindi in crisi la moralità stessa del principio escatologico di ogni religione positiva, dimostrando come esso faccia leva non sulla proclamata autonomia della morale, ma su premio e castigo, dando luogo ad una "morale del ricatto". «La filosofia cerca [...],-- spiega Schopenhauer qui come dappertutto, le delucidazioni vere, ultime, fondate sulla natura dell'uomo, indipendenti da tutte le interpretazioni mitiche, da dogmi religiosi e ipostasi trascendenti, intorno al problema che le sta a cuore, e pretende di vederle provate nell'esperienza esterna ed interiore» ${ }^{4}$.

\footnotetext{
${ }^{3}$ Idem, p. 188.

${ }^{4}$ Idem, p. 207.
} 
Revista Voluntas: Estudos sobre Schopenhauer - Vol. 3, Números 1 e 2 - $1^{\circ}$ e $2^{\circ}$ semestres de 2012 - ISSN: 2179-3786 - pp. 111-121.

3.1 Libertà del volere. La realtà, per Schopenhauer, è generata da una volontà cieca, affamata di vita, «che è costretta a divorare le sue stesse carni $\rangle^{5}$, sovraindividuale, in cerca di continuo piacere, piacere che non può essere mai soddisfatto. La vita, quindi, è contaminata dal dolore già alla sua origine, essa è già una caduta nell'esistenza. In questo senso, il pessimismo metafisico schopenhaueriano è radicale, irrevocabile. Ma è a partire proprio dalla presa d'atto del nonsense della vita, del suo male radicale, della lotta continua per la vita e, soprattutto, della realtà del dolore che Schopenhauer muove per costruire la sua risposta in campo morale. Descrivendo, in via di sintesi, la posizione di Schopenhauer riguardo alla morale, si dovrà fare riferimento necessariamente alla negazione della Libertà del volere umano e al Fondamento della morale. Muovendo da un rigido determinismo nel mondo della natura, Schopenhauer non ammette alcun liberum arbitrium indifferentiae. Anche l'uomo, come il resto degli esseri naturali, sottostà alla legge di causalità ed ogni sua decisione è determinata dal suo carattere, che è innato ed immutabile. L'illusione che conduce gli individui a credere nella propria libertà personale è dovuto al fatto che la scelta degli uomini non è determinata solamente in maniera diretta dalle cause fisiche, come accade nel regno inorganico, o dagli stimoli, come invece accade nel regno vegetale, bensì, come in tutti gli animali, essa è anche mediata dalla motivazione, ovvero da un «causalità che passa attraverso il conoscere» ${ }^{6}$. Mentre però gli animali apprendono esclusivamente in maniera intuitiva, quindi in un 'eterno presente', la conoscenza umana, in virtù della ragione, che Schopenhauer intende come capacità di astrarre concetti universali, determina la percezione del tempo. La prospettiva temporale dell'agire umano allarga il campo di valutazione dell'individuo, che compie le proprie scelte sulla base del maggior vantaggio possibile, presente e futuro. Pertanto, secondo Schopenhauer, l'uomo gode esclusivamente di una libertà relativa alle numerose condizioni esterne, ovvero egli è «libero dalla diretta costrizione degli oggetti concretamente presenti che agiscono da motivi sulla sua volontà». La scelta, però, è sempre determinata con necessità dal carattere individuale e dai fattori esterni, «ognuno sarà prevalentemente spinto da quei motivi, ai quali è accessibile in prevalenza; come un corpo reagisce soltanto agli acidi, l'altro soltanto agli alcali: e né questo né quello può mutare» ${ }^{7}$. L'agente, quindi, è sempre la Volontà universale, che si manifesta nell'uomo attraverso il principium individuationis nel carattere; il reagente, per così dire, è l'esperienza vissuta. La combinazione, per così dire, chimica di questi due fattori, quindi, è sempre determinata in maniera causale necessaria. Quella libertà assoluta, proclamata dagli idealisti Fichte,

\footnotetext{
${ }^{5}$ SCHOPENHAUER, A., Parerga e paralipomena, II, p. 422.

${ }^{6}$ SCHOPENHAUER, A., La libertà del volere umano, p. 75.

${ }^{7}$ SCHOPENHAUER, A., Il fondamento della morale, p. 262.
} 


\section{FABIO CIRACÌ}

Schelling e Hegel, è quindi, per Schopenhauer, solo illusoria. L'unica maniera per cambiare una scelta individuale è opporre ad un tale motivo un contromotivo più forte, così come avviene per le energie, secondo lo schema del parallelogramma delle forze.

3.2 Il carattere dell'uomo. Per il filosofo di Danzica, quello dell'uomo è un carattere innato, immutabile ed individuale, ma esso è anche empirico, ovvero lo si conosce attraverso il suo svolgersi. In questo senso, per Schopenhauer, è valida la massima latina dell'operari sequitur esse, ovvero l'essenza di un carattere si evince esclusivamente dal suo comportamento empirico, dal suo agire concreto, e non è nota a priori né a chi agisce né a terzi. Ad ogni carattere empirico corrisponde però un carattere intelligibile, che è condizione del primo, ma che ci sfugge del tutto e che ci è inconoscibile come ogni cosa in sé, velle non discitur. Se il carattere intelligibile fosse conoscibile, allora «Ogni atto si potrebbe anche predire, persino calcolare con sicurezza». La libertà dell'uomo, quindi, come già per Kant, è solo noumenica. Per Schopenhauer, inoltre, essa è causata dalla volontà, della quale il carattere non è che un'idea, ovvero un grado della sua oggettivazione. Nel mondo dei fenomeni, e perciò in quello degli individui, però, la libertà assoluta non trova posto. Nel mondo della natura, al quale appartiene anche l'uomo, vige esclusivamente il Quidquid fit necessario fit. Libero significa pertanto conforme a volontà in quanto essa è la ragione sufficiente dell'agire ${ }^{8}$.

Ogni carattere si distingue per la presenza, in quantità e qualità differenti, di tre impulsi principali, che determinano tutte le azioni: «In genere esistono soltanto tre impulsi fondamentali delle azioni umane; e solo sollecitando questi impulsi si ha l'influsso di tutti gli altri motivi possibili. Essi sono: $a$. L'egoismo che vuole il bene proprio (ed è sconfinato); $b$. La cattiveria che vuole il male altrui (e arriva fino all'estrema crudeltà); $c$. La compassione che vuole il bene altrui (e arriva fino alla nobiltà d'animo e alla magnanimità» ${ }^{9}$. Quello che Schopenhauer descrive con l'immensa diversità della disposizione innata degli individui»» ${ }^{10}$ altro non è che la «apriorità del carattere morale», ovvero la peculiare miscela dei tre impulsi fondamentali in un individuo. Pertanto, «la bontà morale non viene affatto dalla riflessione $[. .$.$] bensì direttamente dalla volontà,$ la cui costituzione è innata, e in sé non è suscettibile di alcun miglioramento mediante l'educazione» ${ }^{11}$. In brevi, buoni si nasce, non si diventa.

\footnotetext{
${ }^{8}$ Cfr. Idem, p. 57.

${ }^{9}$ SCHOPENHAUER, A., Il fondamento della morale, p. 214.

${ }^{10}$ SCHOPENHAUER, A., Parerga e paralipomena, II, p. 302.

${ }^{11}$ Idem, p. 303.
} 
3.3 Il criterio di moralità. Il maggiore di questi impulsi, nel quale la volontà si manifesta in maniera diretta, è l'egoismo. L'egoismo è il vero centro di gravità dell'animale uomo. L'egoismo è il motore di ogni nostra decisione, lo stimolo predominante: «L'egoismo è colossale e domina il mondo». Che l'egoismo sia dominante e onnipresente, per Schopenhauer, è un fatto evidente del quale si fa quotidianamente esperienza. Il contratto, l'istituzione delle leggi e dello Stato, sono tutti generati dall'esigenza di limitare l'egoismo altrui, sono tutti prodotti dall'hobbesiano bellum omnium contra omnes. Schopenhauer si oppone alla visione idealistica di Hegel, per il quale lo Stato era il rappresentante dello Spirito assoluto nel mondo. Per il Saggio di Francoforte, esso deriva «dalla reciproca paura del reciproco potere, previene le svantaggiose conseguenze dell'egoismo universale fin dove può avvenire per via negativa», «un istituto di protezione».

Se Schopenhauer stabilisce l'immoralità dell'agire umano, egli può però stabilire anche il criterio di un'azione orientata in senso morale: «L'assenza di ogni motivazione egoistica è dunque il criterio di un'azione di valore morale» ${ }^{12}$. Questa è la pietra angolare dell'etica schopenhaueriana. La norma è ricavata, ancora un volta, per via negativa: l'assenza di egoismo stabilisce il valore morale di un'azione e definisce che l'importanza dell'agire morale «può esserci soltanto nella sua relazione con gli altri» ${ }^{13}$. La moralità, quindi, interessa il sovraindividuale, essa è l'oltrepassamento dell'individualità, non l'intersoggettività, come avviene in Kant con l'imperativo assoluto. In questo senso, esistono azioni morali, pur non essendo l'uomo dotato di libertà.

Innanzitutto, Schopenhauer distingue le azioni giuste da quelle morali: la virtù della giustizia, riassunta nella massima del neminem laede, del non ledere a terzi, si distingue da quella della compassione, che implica sempre una attività, in conformità alla massima imo omnes, quantum potes, juva, agisci quanto ti è possibile in favore di altri. Per Schopenhauer, la giustizia ha sempre un carattere negativo e passivo, mentre la compassione sempre un carattere positivo ed attivo. Giustizia e compassioni, quindi, sono per Schopenhauer le due vere virtù cardinali della morale. Ma in che modo agiscono su di noi? Affinché la sofferenza altrui diventi motivo delle mie azioni, ed io possa agire con necessità in favore di qualcuno che soffre, è necessario che «io mi identifichi con lui, vale a dire che tutta la differenza tra me e quell'altro, sulla quale poggia proprio il mio egoismo, sia, almeno fino a un certo punto, annullata». Questa conversione, come la chiama altrove Schopenhauer, non è qualcosa di eccezionale o di fantastico, ma «è reale, e tutt'altro che raro: è il quotidiano fenomeno della compassione, cioè della immediata partecipazione,

\footnotetext{
${ }^{12}$ SCHOPENHAUER, A., Il fondamento della morale, p. 208.

${ }^{13}$ Idem, p. 210.
} 


\section{FABIO CIRACÌ}

indipendente da ogni altro riguardo, alla sofferenza di un altro e con ciò all'impedimento o annullamento di questo dolore, nel quale alla fine consiste ogni soddisfazione, ogni benessere e ogni felicità. Soltanto questa compassione è la base di ogni giustizia spontanea e di ogni genuino amore del prossimo» ${ }^{14}$. La compassione è un fenomeno originario, un avvenimento «stupefacente, anzi misterioso», che l'uomo non può giustificare. Non si può, quindi, dire perché questo miracolo avvenga, né stabilire perché taluni siano più inclini a partecipare della sofferenza altrui ed altri no. La compassione è una virtù innata, così come lo è il carattere. Il fondamento ultimo del fenomeno originario è in attingibile, metafisico, ovvero è radicato nella volontà.

4.1 Prima e seconda navigazione. Per Schopenhauer la volontà è immutabile, solo la conoscenza può cambiare. «Si rischiara la testa, - ribadisce Schopenhauer - ma il cuore non si migliora». L'immutabilità del carattere si ricollega al cosiddetto lato romantico della filosofia di Schopenhauer, al quale appartengono anche il primato della musica e del genio. Ed è proprio al genio che Schopenhauer attribuisce la capacità di strappare il velo di Maja dei fenomeni, e scoprire l'unità del dolore del mondo, il tat twam asi. Ma genio non vuol dire santo. Lo sguardo del genio, cogliendo immediatamente l'essenza del mondo, non è compassionevole, è indifferente, è disinteressato al mondo. Egli contempla esclusivamente le idee, immobili ed eterne, riconoscendole anche nelle cose singole. Non volge l'attenzione al mondo, che brulica di individui che tribolano nel dolore e nel bisogno. Lo sguardo del genio è aristocratico, è distante. Il genio, infatti, è colui che si distacca dalla volontà, che si fa tutto oggetto, attraverso la conoscenza del mondo. "La volontà giunge con la sua oggettivazione, comunque riesca, alla conoscenza di sé, per la quale diviene possibile la sua soppressione, conversione, redenzione». Si tenga presente quanto Schopenhauer scrive a tal proposito nei Supplementi: «Qui ha luogo effettivamente un antagonismo. Quanto più noi siamo consci dell'oggetto, tanto meno lo siamo del soggetto; quanto più invece quest'ultimo occupa la coscienza, tanto più debole e imperfetta è la nostra intuizione del mondo» ${ }^{15}$. Quest'antagonismo fra conoscenza oggettiva e disinteressata ${ }^{16}$ del genio e quella soggettiva e partecipativa dell'uomo compassionevole è fondamentale: la conoscenza ascetica assolve al ruolo di principio di redenzione attraverso il distanziamento, ovvero attraverso la sospensione del soggetto dal mondo fenomenico. Il genio è di fronte alla volontà, è separato da essa. «Con lo svanire della volontà dalla coscienza è in realtà soppressa anche l'individualità, e con questa il suo dolore e la sua

\footnotetext{
${ }^{14}$ Idem, p. 213.

${ }^{15}$ Idem, p. 1688-89.

${ }^{16}$ Cfr. Idem, p. 1709.
} 
Revista Voluntas: Estudos sobre Schopenhauer - Vol. 3, Números 1 e 2 - $1^{\circ}$ e $2^{\circ}$ semestres de 2012 - ISSN: 2179-3786 - pp. 111-121.

miseria ${ }^{17}$. Nel senso inverso, la compassione passa sempre per la partecipazione e poi per l'identificazione col dolore altrui, ovvero con la volontà altrui. Essa è il riconoscimento che la medesima essenza costituisce il mio io e il resto del mondo, che il mio dolore e quello del prossimo hanno la stessa origine. Infine, per vie diverse, la contemplazione e la compassione precedono la negazione della volontà e ne sono la condizione.

Una volta riconosciuta l'unità della volontà nei fenomeni del mondo, avviene quella che Schopenhauer chiama la conversione, ovvero la negazione della volontà metafisica come causa della sofferenza. Sia attraverso la contemplazione estetica, che attraverso la compassione, l'uomo giunge alla negazione della volontà. Tuttavia, esse sono vie diverse e, con lessico schopenhaueriano, antagoniste. La contemplazione avviene attraverso quella conoscenza di genere particolare che è l'intuizione della volontà metafisica del mondo da parte del genio. Il Mitleid, invece, avviene attraverso una trasformazione della volontà, possibile anche all'uomo comune. Come si è già detto, geni e compassionevoli, per Schopenhauer, si nasce. Il carattere ci è dato, ed è immutabile ed innato. Tuttavia, affinché i nostri tre impulsi fondamentali non rimangano in potentia, cioè non rimangano latenti, devono essere sollecitati dall'esperienza, da un reagente. Ciò significa che se l'impulso dell'egoismo, come quello della cattiveria che è il suo complementare, è quello predominante - perché è con esso che la volontà si manifesta direttamente e alla sua massima potenza nell'uomo - l'impulso della compassione, tuttavia, può essere stimolato attraverso l'esperienza diretta del dolore. L'intelletto, cioè, può fornire i motivi necessari per suscitare il sentimento della compassione. Fermo restando, però, che nell'individuo esistano la predisposizione alla compassione. Riprendendo, quindi, una dottrina già insegnata da Platone [Fedro, 99D], Schopenhauer introduce, allora, il tema della "seconda e migliore navigazione", il platonico deuteros plous, opponendola a quella che potremmo definire il próteros plous, "la prima navigazione" del genio. Così Schopenhauer:

Ma se è vero che la [ascesi] vediamo praticare da coloro che sono già giunti alla negazione della volontà, per mantenersi in essa, è anche vero che il dolore in genere, quale viene inflitto dal destino, è una seconda via (il deuteros plous) per arrivare a quella negazione; anzi possiamo supporre che i più vi giungano solo per essa, e che sia il dolore che uno sente egli stesso, non quello meramente conosciuto, quello che produce più spesso la piena rassegnazione, frequentemente solo in prossimità della morte. Giacché solo a pochi basta per ciò la semplice conoscenza, la quale, trapassando il principium individuationis, produce innanzitutto la più perfetta bontà d'animo e l'amore universale per gli uomini, e infine fa loro

\footnotetext{
${ }^{17}$ Idem, p. 1696-7.
} 


\section{FABIO CIRACÌ}

riconoscere tutti i dolori del mondo come i loro propri, per produrre la negazione della volontà ${ }^{18}$.

La dottrina del deuteros plous, totalmente assente negli scritti dell'Etica schopenhaueriana, è però già presente nel Mondo, ovvero in sede di spiegazione metafisica. La seconda navigazione è una vera e propria conversione determinata dall'esperienza diretta e personale del dolore, per via empirica:

Voglio addurre alcune prove di ciò che nel $\S 68$ ho indicato con l'espressione deuteros plous, ossia della negazione della volontà prodotta dal proprio dolore, gravemente sentito, dunque non solo dal far proprio quello altrui e dalla conoscenza da esso mediata della nullità e tristezza della nostra esistenza. Che cosa avvenga nell'intimo dell'uomo in un'elevazione di tal genere e nel processo di purificazione da essa introdotto, si può renderlo comprensibile con ciò che ogni persona sensibile sperimenta nell'assistere ad una tragedia, con cui quello è di natura affine. [...] Su questo processo si fondano tutte le conversioni che trasformano completamente l'uomo, quali le ho descritte nel testo ${ }^{19}$.

In questa maniera, Schopenhauer allarga la possibilità di redenzione dal dolore alla maggioranza degli uomini, attraverso l'esperienza del proprio dolore, inteso come fenomeno della propria essenza metafisica:

Con la denominazione deuteros plous io ho presentato il dolore in certo senso come un surrogato della virtù e della santità; qui però devo profferire l'ardita parola che noi, tutto sommato, abbiamo più da sperare, per la nostra salvezza e redenzione, da ciò che patiamo e facciamo. [...] Se dunque il dolore ha una tale forza salvifica, questa spetterà in grado ancora maggiore alla morte, temuta più di ogni dolore ${ }^{20}$.

In questo senso, Schopenhauer ammette due tipi di possibilità di raggiungere la compassione e quindi la redenzione: la prima dovuta ad una manifesta inclinazione a priori del carattere innato (volontà), che non ha necessità di essere sollecitata dall'esterno; la seconda, quella determinata dall'esperienza del proprio dolore, attraverso il quale il carattere viene sollecitato all'impulso di compassione, prima latente. Senza l'esperienza a posteriori del dolore, in alcuni l'impulso alla compassione rimarrebbe latente, soggiogato dall'egoismo predominante. Il deuteros plous produce, quindi, una vera e propria conversione interiore. Il dolore funge da reagente nei confronti del

\footnotetext{
${ }^{18}$ Idem, p. $756-59$.

${ }^{19}$ Idem, p. 2172.

${ }^{20}$ Idem, p. 2185.
} 
Revista Voluntas: Estudos sobre Schopenhauer - Vol. 3, Números 1 e 2 - $1^{\circ}$ e $2^{\circ}$ semestres de 2012 - ISSN: 2179-3786 - pp. 111-121.

carattere innato, che fa della sofferenza universale un motivo più forte dell'egoismo ed agisce sul carattere con necessità, determinando in senso morale la condotta dell'uomo.

Tutte queste considerazioni - scrive il filosofo dei Parerga e Paralipomena forniscono una spiegazione più particolareggiata della purificazione, indicata nel capitolo precedente con l'espressione deuteros plous, della conversione della volontà e della redenzione, che vengono provocate dai dolori delle vita e sono senza dubbio le più frequenti. Ché questa è la via di quei peccatori che noi tutti siamo. L'altra via, che, per mezzo della sola conoscenza e della successiva appropriazione dei dolori di un intero mondo, porta alla stessa meta, è la via stretta degli eletti, dei santi, e deve quindi considerarsi una rara eccezione ${ }^{21}$.

Probabilmente, in questo modo, Schopenhauer tiene fermi i due lati della sua dottrina, quello romantico e quello illuministico, descrivendo da un lato una "via stretta" per gli eletti e, dall'altro lato, una "via larga" per la maggioranza - ma non per tutti gli uomini. Entrambe le vie conducono però alla redenzione del genere umano attraverso la negazione della volontà di vita. Vero è che attraverso il deuteros plous Schopenhauer assegna al dolore un importante valore sia conoscitivo che morale: esso è strumento di conoscenza dell'unità metafisica della volontà e forza salvifica, in quanto «processo di purificazione, attraverso il quale soltanto, nella maggior parte dei casi, l'uomo è salvato, cioè distolto dall'errore della volontà di vivere» ${ }^{22}$. Il deuteros plous non è una privilegio di pochi eletti, non è virtù esclusiva del genio o del santo, che sono tali perché le loro qualità sono innate. Il dolore è sempre presente, è la vita stessa, ed ogni uomo, volente o nolente, deve fare $\mathrm{i}$ conti con la sofferenza della propria esistenza. Abbandonata definitivamente ogni forma di religione dogmatica e positiva, Schopenhauer recupera, attraverso la dottrina della noluntas, il senso di una religiosità laica, l'Heilsordung ${ }^{23}$, una forma di moralità fondata sulla compassione umana. Per Schopenhauer, infatti, la sofferenza subita è direttamente proporzionata alla possibilità di redenzione, possibilità che è data a quasi tutta l'umanità:

« Il numero dei trappisti regolari è certamente piccolo, invece la metà dell'umanità è fatta di trappisti involontari: povertà, obbedienza, privazione di tutti i piaceri, anzi dei sollievi più necessari - e spesso anche castità coatta e determinata dalla privazione, sono la loro sorte. La differenza è semplicemente che i trappisti lo fanno per libera scelta, metodicamente e senza speranza di miglioramento, mentre invece il primo modo rientra in quello che nei miei capitoli ascetici ho

\footnotetext{
${ }^{21}$ Idem, p. 2187.

${ }^{22}$ Idem, p. 2182-85.

${ }^{23}$ Cfr. Idem, cap. 49.
} 


\section{FABIO CIRACÌ}

chiamato deuteros plous, a produrre il quale la natura dunque ha già provveduto sufficientemente grazie alle basi del suo ordinamento; e tanto più se, ai mali da essa direttamente derivanti, si aggiungono gli altri, dovuti alla perfidia e alla malvagità degli uomini, in guerra e in pace ${ }^{24}$.

\section{Conclusioni}

Quella di Schopenhauer è una fondazione laica dell'etica, che ha per centro l'ammissione dell'egoismo umano e la possibilità di una qualche redenzione attraverso la negazione della volontà. L'unità metafisica del mondo mette fine a gerarchie di ordine spirituale e trascendente, e riunisce sotto la categoria del dolore tutti i viventi. All'uomo è però lasciata l'opportunità di redimersi dalla volontà di vita: attraverso la stretta porta della contemplazione estetica, riservata agli eletti, oppure attraverso la via dell'esperienza del proprio dolore, il deuteros plous, capace di ridestare nella maggior parte degli uomini quel sentimento di compassione che li rende più propriamente umani.

\section{NOTA BIBLIOGRAFICA}

SCHOPENHAUER, Arthur. Il mondo come volontà e rappresentazione, a cura di Sossio Giametta, testo tedesco a fronte. Milano: Bompiani, 2006.

Laterza, $1994^{\mathrm{III}}$. La libertà del volere umano, intr. di Cesare Vasoli. Roma-Bari: . Il fondamento della morale, intr. di Cesare Vasoli, tr. it. di Ervino Pocar. Roma-Bari: Laterza, 2005.

Parerga e paralipomena, 2 voll., vol. I a cura di Giorgio Colli, vol. II a cura di Mario Carpitella, capp. I-XIV tr. di Mazzino Montinari, capp. XV-XXXI tr. di Eva Amendola Kühn. Milano: Adelphi, 2003 ${ }^{\mathrm{II}}$.

CERONETTI, Guido. La lanterna del filosofo. Milano: Adelphi, 2005.

\footnotetext{
${ }^{24}$ SCHOPENHAUER, A., Parerga e paralipomena, II, p. 418.
} 